\title{
Emergence of Agent-based Referral Networks
}

\author{
Bin Yu \\ Department of Computer Science \\ North Carolina State University \\ Raleigh, NC 27695-7535, USA \\ byu@eos.ncsu.edu
}

\author{
Munindar P. Singh \\ Department of Computer Science \\ North Carolina State University \\ Raleigh, NC 27695-7535, USA \\ singh@ncsu.edu
}

\begin{abstract}
We consider the problem of finding desirable services and sources of information in distributed multiagent systems. Traditional solutions, such as centralized directories, may be inaccurate and may often be unavailable in such settings. Therefore, the only way agents can find the right agents is through referrals. In order to make the referrals effective, the agents must adaptively select the agents with whom they interact. This adaptivity leads to the emergence of referral networks among agents. We study this network experimentally to see the emergence of social structures and the evolution of such structures under different scenarios.
\end{abstract}

\section{INTRODUCTION}

The importance of referrals to the effectiveness of social networks has been known for a long time, e.g., [7]. People can usually find the right people to whom to ask something. A celebrated example comes from the experiments of the sociologist Stanley Milgram in the 1960s [4]. Milgram handed letters (addressed to a resident of Boston) to several people in Kansas with the requirement that the letter be passed from person to person until it arrived at its addressee. Surprisingly, it took only about six hops on average for the letters to arrive. This result became well known as six degrees of separation. Milgram's example did not exactly use referrals, although it involved a social network. But it could just as well have been run using referrals.

Agent-based referral systems appeared only recently, e.g., MINDS (based on the documents used by each user) [5], and ReferralWeb (based on co-citations, i.e., co-occurrence of names on WWW pages) [3]. MINDS is the earliest agentbased referral system that we know of. Kautz et al. pioneered the methods of graph analysis for referral systems, in which they find someone with the needed information explicitly using referral chains [2]. Kautz et al. model referral systems statically as a graph and consider referrals directly through path search in the graph without adaptivity. They did not concentrate on the dynamic aspects of social struc-

Permission to make digital or hard copies of all or part of this work for personal or classroom use is granted without fee provided that copies are not made or distributed for profit or commercial advantage and that copies bear this notice and the full citation on the first page. To copy otherwise, to republish, to post on servers or to redistribute to lists, requires prior specific permission and/or a fee.

AAMAS'02, July 15-19, 2002, Bologna, Italy.

Copyright 2002 ACM 1-58113-480-0/02/0007 ...\$5.00. ture. They don't have a learning component for each agent and don't study the dynamics of social structure, especially any emergence or evolution of referral networks due to referrals.

\section{REFERRAL NETWORKS}

Referral networks involve a set of software agents which can automatically generate and follow referrals. We study such networks experimentally to see the dynamics of social structures under different scenarios. Each agent represents the expertise and interests of its (simulated) principal. Simulated queries for information are generated based on the principal's interests. Simulated answers are produced based on the expertise. Each agent also maintains models of some other agents. Some of these agents are considered neighbors. Queries originated by an agent go to selected neighbors; an agent selects the neighbors to contact based on an evaluation of their usefulness for the particular query it is considering. Likewise, when the agent is queried by other agents, it may send back referrals to some of its neighbors. An agent who received referrals may or may not follow up with queries to the referred agents.

To enable the agent to change its neighbors, each agent maintain models of some other acquaintances as well. Acquaintances are not directly queried, but the agent would become acquainted with them when one of its neighbors refers to them. Some acquaintances can get promoted to neighbors if they prove to be useful. To ensure that our simulation is realistic, we require that each agent be limited in the total number of neighbors that it may have. Thus when an acquaintance is promoted to neighbor, typically an neighbor is demoted to a non-neighbor at the same time (but may remain an acquaintance).

The simulated interests of the principal are used to evaluate the quality of the response received from another agent. When an agent provides a high quality response, the querying agent raises the estimated expertise in its model of the provider. The sociability of an agent indicates the effectiveness of the referrals it produces. So when a high quality response is received, the querying agent also raises the estimated sociability of every agent who provided a referral that led to this provider. The agents who had fewer referral hops to the provider are given a higher credit than those who had more referral hops.

We adapt the vector space model (VSM) to locate agents. The VSM was originally applied in modeling documents for retrieval, but it applies here for generating referrals. Each agent maintains a model of its principal and models of its 
acquaintances. The expertise of each user is modeled as an expertise vector. Given a query vector and an expertise vector, the similarity between the two vectors is defined as the cosine of the angle between those vectors.

DeFinition 1. Given a query vector $Q=\left\langle q_{1}, q_{2}, \ldots, q_{n}\right\rangle$ and an expertise vector $E_{P_{i}}=\left\langle e_{1}, e_{2}, \ldots, e_{n}\right\rangle$, the similarity between $Q$ and $E_{P_{i}}$ is defined as:

$$
Q \diamond E_{P_{i}}=\frac{\sum_{t=1}^{n} q_{t} e_{t}}{\sqrt{n \sum_{t=1}^{n}\left(q_{t}\right)^{2}}}
$$

When a user agent receives a query, it tries to match the query against the expertise of its principal. If there is a good match, the query is passed on to the principal directly.

DEFINITION 2. Given a query vector $Q$ and a threshold $\omega_{P_{i}}$, where $0 \leq \omega_{P_{i}} \leq 1$, it says there is a good match between the user $P_{i}$ and the query $Q$ if $Q \diamond E_{P_{i}} \geq \omega_{P_{i}}$.

The relevance of a neighbor to a given query depends not only on the similarity of the query to the neighbor's expertise, but also on the neighbor's sociability, which reflects how much we can trust the referrals produced by this neighbor.

DeFinition 3. Given a query vector $Q$, the relevance of $Q$ and any neighbor $P_{j}$ of the user is computed as $Q \triangle P_{j}=$ $(1-\eta)\left(Q \diamond E_{P_{i}}\right)+\eta S_{j}$, where $S_{P_{j}}$ is the sociability of the neighbor $p_{j}$; and $\eta$ and $(1-\eta)$ are the weights given to sociability and expertise, respectively.

Further, the user may be allowed to specify an absolute relevance threshold. The threshold $\Omega_{P_{i}}$ can be adjusted to tune the number of purported experts found and to limit the number of referrals that user $P_{i}$ will give other users. Note that usually $\Omega_{P_{i}} \geq \omega_{P_{i}}$.

DEFINITION 4. Given a query vector $Q$ and a threshold $\Omega_{P_{i}}$, a neighbor $P_{j}$ of user $P_{i}$ is relevant to $Q$ if $Q \triangle P_{j} \geq \Omega_{P_{i}}$ for a special value of $\eta$.

\section{APPLICATION EXAMPLES}

There are two main application areas for referral networks: knowledge management and distributed reputation management, both of which apply in settings where agents must help each other through referrals to locate desirable agents with whom to interact. The underlying idea is that the agents stand for principals: people, businesses, or (electronic) service providers.

In knowledge management, all the principals are people who interact with each other to share knowledge. Referral networks apply in distributed, dynamic settings and help support effective, natural knowledge management systems. Each user participates in his personal social network [6]. A prototype system MARS is being implemented for use in a practical social network for knowledge sharing.

In distributed reputation management, some of the principals are service providers and some are prospective (business or individual) users of those services. The users help each other identify the best service providers. When evaluating the trustworthiness of a given party, an agent combines its local evidence (based on direct prior interactions with the party) with the testimonies of others regarding the same party through referral networks. In both of these application areas, it would be practically or even conceptually difficult to employ a centralized directory that could produce the right referrals for all comers [1].

\section{CONCLUSION}

Referral networks can be applied in building multiagent systems in general [8]. The conventional way to implementing a multiagent system is to use specialized agents such as brokers or facilitators. Despite considerable research that has gone into the theories and architectures for such multiagent systems, there is relatively little experience with building a multiagent system without centralized directories. A referral network approach, being perfectly decentralized, would not only be more resistant to failure but would also lead to the dissemination of better vetted information.

In future work, we plan to continue to explore other aspects of the social structures that arise in referral networks and in particular to relate these structures to quality of referral networks. Lastly, we plan to study the efficiency of referral networks using a bibliography of artificial intelligence publications. We will focus on how to model the whole process of referral chaining and how to improve the accuracy of referral chains.

\section{ACKNOWLEDGEMENTS}

This research was supported by the National Science Foundation under grants IIS-9624425 (Career Award) and ITR0081742. We are indebted to the anonymous reviewers for their helpful comments.

\section{REFERENCES}

[1] K. Decker, K. Sycara, and M. Williamson. Middle-agents for the internet. In Proceedings of the International Joint Conference on Artificial Intelligence (IJCAI), pages 578-583, 1997.

[2] H. Kautz, B. Selman, and A. Milewski. Agent amplified communication. In Proceedings of the National Conference on Artificial Intelligence, pages 3-9, 1996.

[3] H. Kautz, B. Selman, and M. Shah. The hidden web. AI Magazine, 18(2):27-36, 1997.

[4] S. Milgram. The small world problem. Psychology Today, 2:60-67, 1967.

[5] U. Mukhopadhyay, L. Stephens, M. Huhns, and R. Bonnell. An intelligent system for document retrieval in distributed office environments. Journal of American Society for Information Sciences, 37:123-135, 1986.

[6] B. A. Nardi, S. Whittaker, and H. Schwarz. It's not what you know, it's who you know: work in the information age. First Monday, 5, 2000.

[7] P. H. Reingen and J. B. Kernan. Analysis of referral networks in marketing: Methods and illustration. Journal of Marketing Research, 23:370-378, Nov. 1986.

[8] M. P. Singh, B. Yu, and M. Venkatraman. Community-based service location. Communications of the ACM, 44(4):49-54, 2001. 